\title{
Depression, post-traumatic stress, anxiety, and fear of COVID-19 in the general population and health-care workers: prevalence, relationship, and explicative model in Peru
}

David Villarreal-Zegarra ${ }^{1,2}$, Anthony Copez-Lonzoy ${ }^{2,3,4^{*}}$, Ana L. Vilela-Estrada ${ }^{2,5}$ and Jeff Huarcaya-Victoria ${ }^{6,7}$

\begin{abstract}
Background: This study has two aims. First, determine the fit of the fear model to COVID-19, anxiety, and posttraumatic stress in the general population and health-care workers. Second, determine which model best explains the relationship between depression and the triad of fear, anxiety, and post-traumatic stress in both groups.

Method: A cross-sectional study was conducted using self-reported questionnaires for anxiety, fear of COVID-19, depression, and post-traumatic stress. Information was collected from adults living in Lima, the capital and the most populous city in Peru. The explanatory models were evaluated using a structural equation model.

Results: A total of 830 participants were included, including general population $(n=640)$ and health-care workers $(n=190)$. A high overall prevalence of depressive symptoms $(16 \%)$, anxiety $(11.7 \%)$, and post-traumatic stress (14.9\%) were identified. A higher prevalence of depressive, anxious, or stress symptoms was identified in the general population (28.6\%) compared to health-care workers (17.9\%). The triad model of fear of COVID-19, anxiety, and stress presented adequate goodness-of-fit indices for both groups. A model was identified that manages to explain depressive symptoms in more than $70 \%$ of the general population and health-care workers, based on the variables of the triad $(\mathrm{CFI}=0.94 ; \mathrm{TLI}=0.94 ; \mathrm{RMSEA}=0.06 ; \mathrm{SRMR}=0.06)$. In the general population post-traumatic stress mediated the relationship between anxiety and depression $(\beta=0.12 ; 95 \% \mathrm{Cl}=0.06$ to 0.18$)$ which was significant, but the indirect effect of post-traumatic stress was not significant in health care workers $(\beta=0.03$; $95 \% \mathrm{Cl}=-0.11$ to 0.19 ).
\end{abstract}

Limitations: The prevalence estimates relied on self-reported information. Other variables of interest, such as intolerance to uncertainty or income level, could not be evaluated.

\footnotetext{
* Correspondence: anthonycopez22@gmail.com

${ }^{2}$ Instituto Peruano de Orientación Psicológica, Lima, Peru

${ }^{3}$ Unidad de Investigación en Bibliometría, Universidad San Ignacio de Loyola, Lima, Peru

Full list of author information is available at the end of the article
}

(C) The Author(s). 2021 Open Access This article is licensed under a Creative Commons Attribution 4.0 International License, which permits use, sharing, adaptation, distribution and reproduction in any medium or format, as long as you give appropriate credit to the original author(s) and the source, provide a link to the Creative Commons licence, and indicate if changes were made. The images or other third party material in this article are included in the article's Creative Commons licence, unless indicated otherwise in a credit line to the material. If material is not included in the article's Creative Commons licence and your intended use is not permitted by statutory regulation or exceeds the permitted use, you will need to obtain permission directly from the copyright holder. To view a copy of this licence, visit http://creativecommons.org/licenses/by/4.0/ The Creative Commons Public Domain Dedication waiver (http://creativecommons.org/publicdomain/zero/1.0/) applies to the data made available in this article, unless otherwise stated in a credit line to the data. 
Conclusions: Our study proposes and tests one model that explains more than $70 \%$ of depressive symptoms. This explanatory model can be used in health contexts and populations to determine how emotional factors can affect depressive symptoms.

Keywords: Depression, Post-traumatic stress, Anxiety, Fear of COVID-19, Peru

\section{Background}

Peru is one of the countries most affected worldwide by the COVID-19 pandemic. According to the official data given by the Peruvian Government, up to June 06, 2021, there have been 1,983,570 confirmed cases and 186,511 deaths, and the mortality rate was $9.40 \%$ [1]. To ensure a decrease in the spread of the disease, policies, such as isolation and quarantine, have been taken to limit contact and exposure [2]. For instance, on March 16, 2020, Peruvian authorities decreed a state of emergency with mandatory quarantine measures.

The COVID-19 pandemic and the quarantine measures have generated different social and economic problems, which, added to the fear of catching the virus, have affected the mental health of the general population. The prevalence of stress, anxiety, and depression among the general population during the COVID-19 pandemic is estimated to be $29.6,31.9$, and $33.7 \%$, respectively [3]. These figures represent an increase in the prevalence of these mental health disorders compared to pre-pandemic measurements in the general population [3]. Evidence suggests that individuals who have been isolated and quarantined due to COVID-19 have experienced significant levels of anxiety, anger, confusion, and stress [4]. Also, fear of COVID-19 is associated with the presence of anxious depressive symptoms and post-traumatic stress [5].

Fear is one of the most influential factors in the presence of emotional problems, such as anxiety and stress. Evidence from animal models of fear and human studies indicates that exposure to constant fear increases anxiety, which in turn can trigger traumatic stress [6-9]. One possible explanation is that the endocannabinoid system links the perception of external and internal stimuli with different neurophysiological and behavioral outcomes, such as the reaction to fear, anxiety, and stress. This neurobiological mechanism allows the subject to adapt or not to this stress. A traumatic event or a highly stressful situation (i.e. a pandemic) could trigger the emergence of traumatic stress if the fear and anxiety response is not adaptive [7]. Therefore, this relationship can be understood as a sequential process of fear, anxiety, and post-traumatic stress, considering that fear also directly influences the appearance of stress.

\section{Hypothesis a: triad of fear, anxiety, and post-traumatic stress (see Fig. 1a)}

This triad involves short-term or medium-term emotional responses such as the fear and anxiety response to a highly stressful situation (i.e., a pandemic). Fear is defined as the fear or aversion response to a concrete, known or defined situation (i.e., the possibility of catching the virus) [10]. In contrast, the anxiety response is a non-specific, diffuse sensation that is not necessarily associated with concrete or well-defined element (i.e., the context of the pandemic) [10]. Fear, anxiety, and a highly stressful event are elements that correspond to the fear circuit that could trigger acute stress and subsequent post-traumatic stress [10]. Acute stress usually begins immediately after the highly stressful event and lasts from 3 days to 1 month; however, post-traumatic stress can manifest as early as the first month after the event. Therefore, both persons directly exposed to the virus (i.e., healthcare workers) and persons exposed but not exposed but anxious about the uncertainty of the future, the possible infection of family members, and the avalanche of news about COVID-19 (i.e., the general population and healthcare workers) [11], could trigger a state of post-traumatic stress disorder resulting from a highly stressful event such as facing the first few months of the pandemic.

Although there is ample evidence about the triad of fear, anxiety, and post-traumatic stress, it is not understood how this triad influences the presence of depressive symptoms. Fear of COVID-19 is weakly related to depressive symptoms but more strongly related to anxiety and post-traumatic stress [5]. However, there are several possibilities as to how depression can be explained by the triad of fear, anxiety, and post-traumatic stress (see Fig. 1b-e). Therefore, this study raises four possible hypotheses (hypotheses B, C, D, and E) that could explain the role of depression within this relationship.

\section{Hypothesis B: post-traumatic stress influences depression (see Fig. 1b)}

In many cases, post-traumatic stress is not the only condition resulting from traumatic experiences (i.e., living in a pandemic), but also the onset of other comorbid conditions, including depression, somatization, or physical problems [12]. Longitudinal studies have identified that post-traumatic stress predicts depression $[13,14]$, so the hypothesis that post-traumatic stress influences depression is plausible, considering that this stress is preceded by the fear of COVID-19 and by anxious symptoms [5]. 


\section{Relationship of fear, anxiety, and PTSD}

A)

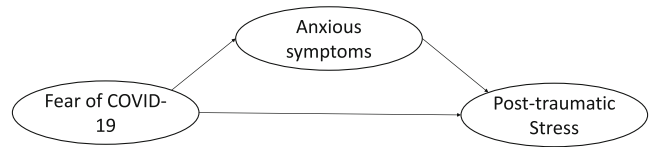

Framework explaining Depression
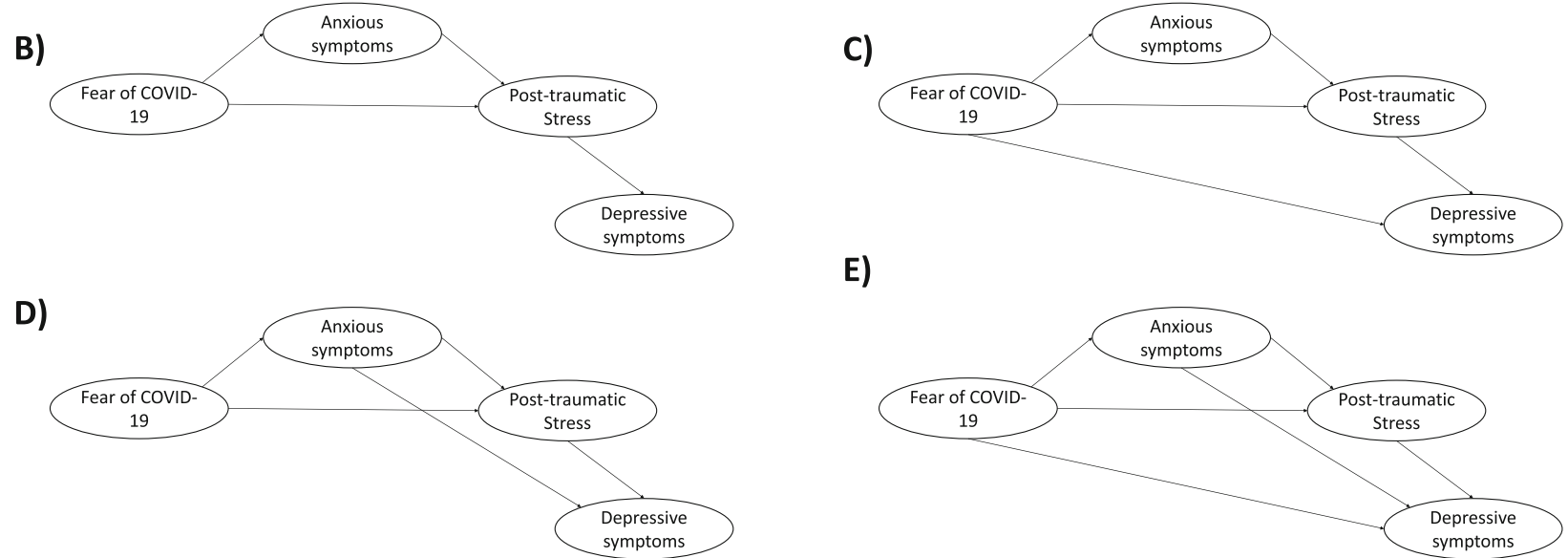

E)

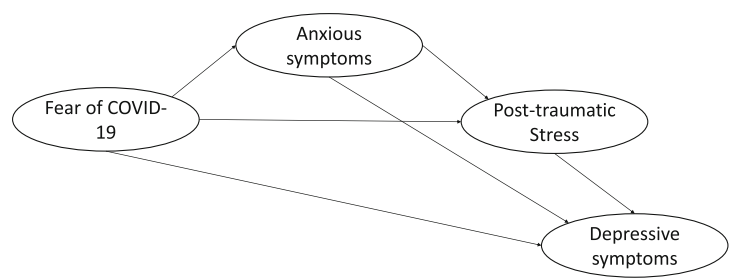

Fig. 1 Models that explain the relationship between depression, fear, anxiety, and post-traumatic stress. Note: Figure 1a Hypothesis A: Triad of fear, anxiety, and post-traumatic stress. Figure 1b Hypothesis B: Post-traumatic stress influences depression. Figure 1c Hypothesis C: Fear of COVID-19 influences depression, while anxiety and post-traumatic stress are mediators. Figure 1d Hypothesis D: Anxiety influences depression and post-traumatic stress is a mediator. Figure 1e Hypothesis E: Fear, anxiety, and post-traumatic stress are related to depression

Hypothesis C: fear of COVID-19 influences depression, while anxiety and post-traumatic stress are mediators (see Fig. 1c)

This model assumes that the anxious and depressive symptoms are not directly related, but together with post-traumatic stress, they are mediators. However, there is abundant evidence that anxiety and depression are closely related $[15,16]$. It is feasible that the force of the relation between anxiety and depression diminishes if post-traumatic stress acts like a mediator [17]. This could support the hypothesis, but it is necessary to prove it with data.

\section{Hypothesis D: anxiety influences depression and post- traumatic stress is a mediator (see Fig. 1d)}

In hypothesis $\mathrm{B}$, the relationship between post-traumatic stress and depression was justified, but in this model, as evidence indicates, post-traumatic stress is considered to be a mediating factor in the relationship between anxiety and depression [18]. Some studies have also identified that post-traumatic stress can mediate depression with other mental health problems. Studies in refugees exposed to different forms of trauma indicate the mediating effect of post-traumatic stress on some mental disorders, such as depression, substance abuse, and personality disorders [19]. In addition, post-traumatic stress can also act as a partial mediator of the relationships between trauma and the severity of depression and between trauma and general mental functioning [20]. Likewise, there is abundant evidence that anxious and depressive symptoms are strongly correlated with each other [15], even in the context of COVID-19 [16]. Therefore, it is suggested that this may be a tentative model of the relationship between depression with fear, anxiety, and post-traumatic stress. For our study, we considered the mediating role of post-traumatic stress since the evidence suggests that this variable behaves as a mediator and not as a moderator.

Hypothesis E: fear, anxiety, and post-traumatic stress are related to depression (see Fig. 1e)

This model assumes the same assumptions as to the previous hypothesis (model D) but considers that fear of COVID-19 and depression are directly related. It should be noted that although fear of COVID-19 and depression in bivariate analyses have found a positive but low correlation [5], this correlation may increase if mediated by factors such as anxiety or post-traumatic stress. Additionally, by considering all possible relationships, it is possible to see more clearly which dimensions are more or less related. 
A better understanding of the relationship of the triad of fear, anxiety, and post-traumatic stress with the appearance of depressive symptoms will allow the identification of how these variables would trigger emotional problems during the context of the COVID-19 pandemic. An important element to consider is that the model could be affected if the groups evaluated have a higher exposure to or knowledge about the virus. Therefore, it is necessary to identify whether the models evaluated are equivalent among health-care workers and the general population. Because the former has greater knowledge of the treatment and evolution of the virus and greater average exposure to the virus due to the nature of their work, the relationships between the variables of the triad with depressive symptoms could be affected.

Therefore, there are two objectives related to the previously presented hypotheses. The first is to determine if "hypothesis A" about the relationship between the fear of COVID-19, anxiety, and post-traumatic stress fits adequately with the data collected from the general population and health-care workers (see Fig. 1a). The second is to determine which of the hypotheses presented above best explains the relationship between depression and the triad of fear, anxiety, and post-traumatic stress in both groups (see Fig. 1b-e). Our study considers a differentiated assessment of the general population and healthcare workers as they are exposed to different concrete and unspecific conditions of fear, anxiety, and stress.

\section{Methods}

\section{Study design}

A cross-sectional study was conducted that included the use of self-reported questionnaires. An online survey was used to avoid physical contact and the spread of SARS-CoV-2 among participants. Demographic and social data of the participants were obtained. Anxiety, fear of COVID-19, depression, and post-traumatic stress were measured using validated questionnaires and scoring systems. Data collection took place over 1 week, April 17-23, 2020, 1 month after the state of emergency was declared and mandatory self-quarantine was ordered in Peru. The sampling was not probabilistic and we used networks of contacts through social networks and other digital media to circulate the online survey.

\section{Participants}

Information was collected from adults living in Lima, the capital of Peru and the most populous city in the country. Inclusion criteria included: 18 to 80 years of age and an agreement to participate in the online survey. The participants were divided into two groups: the general population and health-care workers. Data collection sought to provide a sufficient number of cases from each group to perform the analyses (at least 150 participants per group) [21].

\section{Variables and measurement instruments Fear of COVID-19}

The Fear of COVID-19 Scale (FCV-19S) is a onedimensional scale with seven items used to assess fears of COVID-19 in the general population. The items are scored on a 5-point scale ranging from 1 (strongly disagree) to 5 (strongly agree). Total scores range from 7 to 35 , indicating that, with higher scores, fear of COVID-19 is increased [22]. The reliability values of the scores for internal consistency were acceptable to $\alpha=0.82$. The evaluation properties of the instrument have been evaluated in a previous study [5]. There is evidence of validity and reliability in their scores.

\section{Symptoms of post-traumatic stress}

The Impact of Event Scale-Revised (IES-R) was used, with 22 items scored with a five-point scale, ranging from 0 (none) to 4 (extremely) [23]. The IES-R is a selfreport scale of three dimensions: a) intrusion dimension which evaluates indicators of intrusive thoughts, nightmares, intrusive feelings and images, and a new dissociative-type experience (item 1, 2, 3, 6, 9, 14, 16, and 20); b) avoidance dimension which is used to evaluate indicators of numbness and avoidance of feelings, situations, and ideas (item 5, 7, 8 11, 12, 13, 17, and 22); c) hyperarousal dimension which analyzes indicators of anger, irritability, hypervigilance, difficulty concentrating, and intensified startle response (item 4, 10, 15, 18, 19, and 21). This instrument has shown good internal consistency $(\alpha=0.964)$. The three dimensions are summed and present an overall score with a cohort point of 33 or more points corresponding to posttraumatic stress symptoms [24]. The premise used to define the highly stressful IES-R event was "respond based on your experience of the COVID-19 pandemic.

\section{Depressive symptoms}

The Patient Health Questionnaire-9 (PHQ-9) was used to evaluate depressive symptoms. The PHQ-9 is a selfadministered scale, scored from 0 (nothing) to 3 (almost every day), that consists of nine items based on the nine indicators of major depression from the DSM-IV [25]. Their scores range from 0 to 27, with a cohort point of 10 being considered as the presence of clinically relevant depressive symptoms [26]. The validation of the PHQ-9 conducted in Peru has shown adequate levels of reliability and validity for a single-dimensional model of the PHQ-9 [27]. 


\section{Anxious symptoms}

The Generalized Anxiety Disorder Scale-7 (GAD-7) is a valid and effective self-reporting instrument for assessing the severity of anxiety disorders in clinical practice [28]. A cohort point of 10 or more is considered to correspond to clinically relevant anxious symptoms [29]. The scale has been previously translated into Spanish and validated [30]. It consists of seven items designed to measure the symptomatology of anxiety during the 2 weeks before self-application. Each item is scored on a Likert scale ranging from 0 (nothing) to 3 (almost every day). In the present study, the GAD-7 had adequate internal consistency (Cronbach's alpha $=0.898$ ). Generalized anxiety was assessed, rather than anxiety related to COVID-19, since it corresponds to a global anxiety response (i.e., job loss, illness, family problems) and not only related to the virus infection.

\section{Socio-demographic characteristics}

Socio-demographic information was collected on the following: sex (man or woman), civil status (married, divorced, single, or widowed), educational level (primary, secondary, technical, or university), employment status (formal employment, informal employment, or unemployed), if they profess a religion (yes/no), and if they self-report having a mental health problem (yes/no). Age was recorded as a continuous variable and categorized into six groups of 10 years each $(18-19,20-29,30-39$, $40-49,50-59$, and 60 or more). Additionally, the type and number of COVID-19 symptoms were considered for the creation of a variable based on whether the person reported having a cough, tiredness, muscle pain, headache, or diarrhea. This data was considered within the collection of information concerning the fact that previous research has considered the presence and severity of covid symptoms as a characteristic that can favor the appearance of reaction levels of emotional distress, stress, anxiety, depression, and PTSD [31, 32] as populations with worse health conditions may experience greater psychological vulnerability due to uncertainty about their health status, follow-up, treatment and care [3].

\section{Procedures}

Data collection focused on collecting a sufficient number of participants for the general population and health professionals, so the questionnaire was socialized through social networking groups specific to health workers and through profile pages where there is a greater reach to the general population.

\section{Data analysis}

\section{Descriptive and prevalence}

A descriptive analysis was conducted for the general population and health-care workers. Also, the prevalence of clinically relevant depressive symptoms (PHQ-9 $\geq 10$ points) [26], clinically relevant anxious symptoms (GAD-7 $\geq 10$ points) [29], and post-traumatic stress symptoms (IES-R $\geq 33$ points) [24] was analyzed.

\section{Relation between variables and reliability}

The correlation between variables was determined by Spearman $\left(r_{s}\right)$ since the normality assumptions were not fulfilled. A large $\left(r_{s}>0.70\right)$, moderate $\left(r_{s}>0.50\right)$, or small $\left(r_{s}>0.30\right)$ ratio is determined based on the size of the correlation coefficient. In addition, reliability was evaluated with the omega coefficient, considering adequate values greater than 0.80 [33].

\section{Structural equation model}

A structural equation model was used using the weighted least squares means and variance adjusted (WLSMV), which allows handling non-normality [34]. This analysis used the polychoric matrices suitable for the ordinal nature of the items $[35,36]$. All of the models presented in Fig. 1 were evaluated. First, we evaluated a baseline model that supports the relationship between fear of COVID-19, anxiety, and post-traumatic stress (model A) to have evidence that it is a model with adequate adjustment based on which to evaluate the more complex models. This model was taken as a baseline because it presents sufficient evidence to support it, as described in the background section. Then models $1 \mathrm{~B}$ through $1 \mathrm{E}$ were evaluated to see which model had the best fit and most variance.

It should be noted that the model was adjusted using two socio-demographic variables (sex, age, and symptoms of COVID-19). First, sex was added to the model to influence fear of COVID-19 since the fear of the COVID-19 scale and is not invariant between men and women [5]. Thus, the sex and age variables were added so that the model could be adjusted. Second, anxiety symptoms were adjusted by the COVID-19 symptoms because there are other instruments focused on measuring anxiety-related to COVID-19 that have demonstrated that the anxiety experienced is directly related to the perception of COVID-19 symptoms (i.e., cough, dizziness, muscle pain) [37]. Therefore, it was considered necessary to include these three socio-demographic variables in the model analysis.

Two criteria were used to evaluate the different models. First, different goodness-of-fit indices were evaluated. We used the Comparative Fit Index (CFI) and the Tucker-Lewis Index (TLI), both with appropriate values $\geq 0.90$. The Standardized Root Mean Square Residual (SRMR) and the Root Mean Square Error of Approximation (RMSEA) with a confidence interval of $90 \%$ and with adequate values $<0.08$ were used to compare model fit $[34,38]$. Second, the $R^{2}$ of the outcome variable 
(depressive symptoms) was evaluated, which allows us to know how much variance explains the proposed model. The models that explain the most variance are the most adequate [17].

In addition, mediation analysis assessed the indirect effect of posttraumatic stress on the anxiety-depression relationship. The cross-product of the coefficients was calculated to obtain an indirect effect of the structural model with 5000 iterations were used to calculate the standard error and obtain the path of the indirect effect.

\section{Statistic software}

All analyses were done in $\mathrm{R}$ Studio, with the packages "lavaan," "semTools," and "semPlot."

\section{Ethics}

The study protocol and the instruments used for the evaluation were approved by the ethics committee of the Universidad San Martin de Porres (Oficio No. 2272020-CIEI-FMH-USMP).

\section{Results}

\section{General characteristics and prevalence}

The characteristics of the participants are found in Table 1. The average age of health workers was 38.8 $(\mathrm{SD}=11.2)$ and of the general population was 38.3 (SD = 13.2). The majority of participants were women. The overall prevalence for health-care workers and the general population was $16 \%$ for clinically relevant depressive symptoms, $11.7 \%$ for clinically relevant anxious symptoms, and $14.9 \%$ for post-traumatic stress. It was found that $23.1 \%$ of the participants had one of these mental health problems. A higher prevalence of depressive, anxious, or stress symptoms was identified in the general population (28.6\%) compared to health-care workers (17.9\%).

\section{Relationship between variables and reliability}

The relationship between depressive and anxious symptoms was high and very similar in the general population and health-care workers $\left(r_{s}>0.70\right)$. The general population presented a strong relationship between anxious symptoms and post-traumatic stress (overall score and three dimensions), while health-care workers reached a moderate relationship $\left(r_{s}>0.50\right)$ [39]. However, these values did not change much (see Table 2 ). All the instruments evaluated presented adequate levels of internal consistency.

A moderate relationship $\left(r_{s}>0.50\right)$ was found between post-traumatic stress (overall score and three dimensions) with fear of COVID-19 and depressive symptoms for both the general population and health workers. It should be noted that a small relationship was found between fear of COVID-19 and depressive and anxious symptoms in both groups $\left(r_{s}>0.30\right)$.

\section{Structural equation model}

The "model A" or baseline model of the relationship of fear of COVID-19, anxiety, and post-traumatic stress showed adequate goodness-of-fit indices (see Table 3), indicating that this model is stable and could be used to evaluate more complex models to explain depressive symptoms in health-care workers and the general population. Therefore, models to explain depressive symptoms were evaluated using "model A" as a basis (see Fig. 1b-e).

It was identified that the four models evaluated (Model $\mathrm{B}$ to $\mathrm{E})$ identified adequate goodness-of-fit indices in all cases. However, the models that most explained the depressive symptoms were model D (explaining $73.9 \%$ of the variance) and model E (explaining $74.8 \%$ of the variance). Although model $\mathrm{E}$ contributed $0.7 \%$ more variance, the relationship between fear of COVID-19 and depressive symptoms was -0.104 . This finding would be contrary to what is theoretically expected since it is estimated that the greater the fear of the COVID-19, the greater the levels of depressive symptoms are expected, as was found in the bivariate analysis, where these variables presented a direct, significant, and positive correlation (see Table 2). Therefore, it was decided that model $\mathrm{D}$ would best explain the depressive symptoms because it presents sufficient goodness-of-fit indices, explains almost the same amount of variance as model E, and fits best with the theoretical assumptions (see Fig. 2).

Analysis of model D for the total number of participants, the general population, and healthcare workers (see Fig. 2 and Fig. 3), identified that fear of COVID-19 is directly associated with anxiety $(p<0.001)$, and with the post-traumatic stress response $(p<0.001)$. Also, anxiety is directly associated with post-traumatic stress and depressive symptoms $(p<0.001)$. When the relationships between the variables in model $\mathrm{D}$ were evaluated by type population, it was found that the general population and health professionals had very similar values between the variables (see Fig. 3). However, some variables are no longer significant between the two groups. On the one hand, age in health professionals does not associate with anxiety levels $(\beta=-0.065 ; p=0.479)$, while in the general population it does $(\beta=-0.337 ; \mathrm{p}<0.001)$. On the other hand, post-traumatic stress is not associated with the presence of depressive symptoms in the general population $(\beta=0.122 ; p=0.025)$, but not in healthcare professionals where it presents a very small and nonsignificant coefficient $(\beta=-0.015 ; p=0.876)$.

Mediation analysis identified that in the overall participants the indirect effect of post-traumatic stress on the relationship of anxiety and depression was significant 
Table 1 Socio-demographic characteristics $(n=830)$

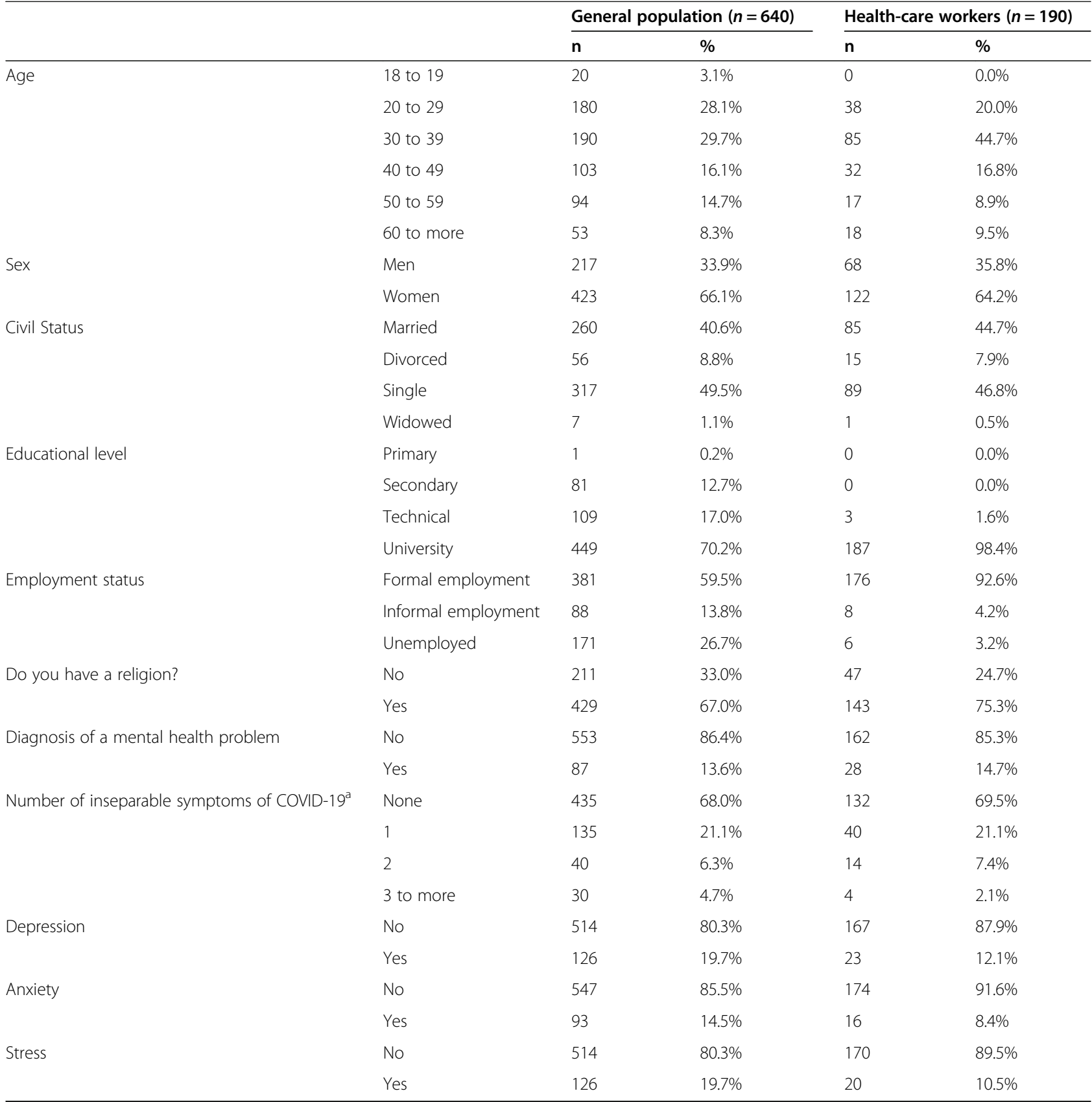

${ }^{a}$ Cough, fatigue, muscle pain, headache, or diarrhea

( $\beta=0.10 ; 95 \% C I=0.05$ to 0.16 ). However, the results varied depending on the population assessed. In the general population, post-traumatic stress mediated the relationship between anxiety and depression $(\beta=0.12 ; 95 \% \mathrm{CI}=$ 0.06 to 0.18 ), but the mediation was not significant in health care workers ( $\beta=0.03 ; 95 \% \mathrm{CI}=-0.11$ to 0.19 ).

A sub-analysis was conducted to assess the role of other sociodemographic variables such as sex and age with COVID-19 fear, anxiety, and post-traumatic stress variables. It was identified that only significant relationships were between fear of COVID-19 and age; post-traumatic stress and sex; and age and anxious symptoms.

\section{Discussion}

Main findings and significance of the results

Our study proposes and tests different models based on structural equations models that allowed testing hypothetical models based on substantive theories that use the triad of fear, anxiety, and post-traumatic stress to explain depressive symptoms in the general population 
Table 2 Relationship between the fear of COVID-19, depressive symptoms, anxious symptoms, and post-traumatic stress ( $n=830$ )

\begin{tabular}{|c|c|c|c|c|c|c|c|c|c|}
\hline & & $(1)$ & $(2)$ & (3) & (4) & $(4.1)$ & $(4.2)$ & (4.3) & $\omega$ \\
\hline \multirow{7}{*}{$\begin{array}{l}\text { General population } \\
(n=640)\end{array}$} & (1) Fear of COVID-19 & 1 & & & & & & & 0.90 \\
\hline & (2) Depressive symptoms & 0.32 & 1 & & & & & & 0.88 \\
\hline & (3) Anxious symptoms & 0.44 & 0.73 & 1 & & & & & 0.89 \\
\hline & (4) Post-traumatic Stress & 0.54 & 0.65 & 0.73 & 1 & & & & $0.98^{\mathrm{a}}$ \\
\hline & (4.1) Intrusion & 0.55 & 0.63 & 0.71 & 0.94 & 1 & & & 0.82 \\
\hline & (4.2) Avoidance & 0.51 & 0.56 & 0.64 & 0.95 & 0.83 & 1 & & 0.93 \\
\hline & (4.3) Hyperarousal & 0.49 & 0.67 & 0.74 & 0.92 & 0.86 & 0.80 & 1 & 0.91 \\
\hline \multirow{7}{*}{$\begin{array}{l}\text { Health-care workers } \\
(n=190)\end{array}$} & (1) Fear of COVID-19 & 1 & & & & & & & 0.91 \\
\hline & (2) Depressive symptoms & 0.35 & 1 & & & & & & 0.87 \\
\hline & (3) Anxious symptoms & 0.48 & 0.74 & 1 & & & & & 0.90 \\
\hline & (4) Post-traumatic Stress & 0.61 & 0.56 & 0.64 & 1 & & & & $0.98^{\mathrm{a}}$ \\
\hline & (4.1) Intrusion & 0.59 & 0.54 & 0.62 & 0.93 & 1 & & & 0.79 \\
\hline & (4.2) Avoidance & 0.57 & 0.46 & 0.55 & 0.94 & 0.80 & 1 & & 0.93 \\
\hline & (4.3) Hyperarousal & 0.54 & 0.64 & 0.69 & 0.91 & 0.84 & 0.77 & 1 & 0.87 \\
\hline
\end{tabular}

The relationship was evaluated with Spearman's coefficient. All values are significant $(p<0.001) .{ }^{\text {a }}$ Omega coefficient considering second-order models

and health-care workers during the COVID-19 pandemic.

We found that this triad, along with three additional variables (number of COVID-19 symptoms, age, and sex), explained more than $71 \%$ of depressive symptoms in the general population and health care workers. The proposed model (model D) allows us to understand the role of fear of COVID-19, age, and the number of perceived COVID-19 symptoms in the presence of anxiety symptoms. In turn, it is found that anxiety symptoms and sex affect the stress symptoms, and all of these are associated with depressive symptoms. Anxiety symptoms are the most influential in the occurrence of depressive symptoms, in compassion to stress. It was identified that the model presents a different behavior for the general population and health-care workers since in the latter group the variables of age and post-traumatic stress are not associated with depressive symptoms.

This explanatory model can be used in health contexts and populations for how emotional factors (fear, anxiety, and stress) can affect depressive symptoms. Also, the model can be used to understand a differential response

Table 3 Goodness-of-fit indices of the structural equation model $(n=830)$

\begin{tabular}{lllllllll}
\hline & & $\boldsymbol{X}^{\mathbf{2}}$ & $\boldsymbol{d f}$ & CFI & TLI & RMSEA [90\% Cl] & SRMR & $\boldsymbol{R}^{\mathbf{2}}$ \\
\hline $\begin{array}{llllll}\text { Both groups }(n=830) \\
\text { Model A }\end{array}$ & 3389.7 & 692 & 0.940 & 0.945 & $0.068[0.066-0.071]$ & 0.056 & - \\
& Model B & 4821.9 & 1069 & 0.923 & 0.928 & $0.065[0.063-0.067]$ & 0.065 & 0.613 \\
& Model C & 4741.9 & 1068 & 0.924 & 0.930 & $0.064[0.062-0.066]$ & 0.063 & 0.682 \\
& Model D & 4120.2 & 1068 & 0.937 & 0.942 & $0.059[0.057-0.061]$ & 0.056 & 0.739 \\
& Model E & 4114.4 & 1067 & 0.937 & 0.942 & $0.059[0.057-0.061]$ & 0.055 & 0.748 \\
General population & Model A & 2674.9 & 692 & 0.946 & 0.951 & $0.067[0.064-0.070]$ & 0.058 & - \\
$(n=640)$ & Model B & 3716.9 & 1069 & 0.933 & 0.938 & $0.062[0.060-0.064]$ & 0.065 & 0.627 \\
& Model C & 3672.3 & 1068 & 0.934 & 0.939 & $0.062[0.060-0.064]$ & 0.064 & 0.689 \\
& Model D & 3262.0 & 1068 & 0.945 & 0.949 & $0.057[0.054-0.059]$ & 0.057 & 0.750 \\
& Model E & 3262.5 & 1067 & 0.945 & 0.949 & $0.057[0.055-0.059]$ & 0.057 & 0.759 \\
Health-care workers $(n=190)$ & Model A & 1196.5 & 692 & 0.956 & 0.960 & $0.062[0.056-0.068]$ & 0.070 & - \\
& Model B & 1859.8 & 1069 & 0.936 & 0.941 & $0.062[0.057-0.067]$ & 0.089 & 0.555 \\
& Model C & 1807.4 & 1068 & 0.940 & 0.945 & $0.060[0.055-0.065]$ & 0.086 & 0.705 \\
& Model D & 1664.6 & 1068 & 0.952 & 0.955 & $0.054[0.049-0.059]$ & 0.078 & 0.716 \\
& Model E & 1662.7 & 1067 & 0.952 & 0.955 & $0.054[0.049-0.059]$ & 0.078 & 0.723 \\
\hline
\end{tabular}




\section{Relationship of fear, anxiety, and PTSD (model A)}

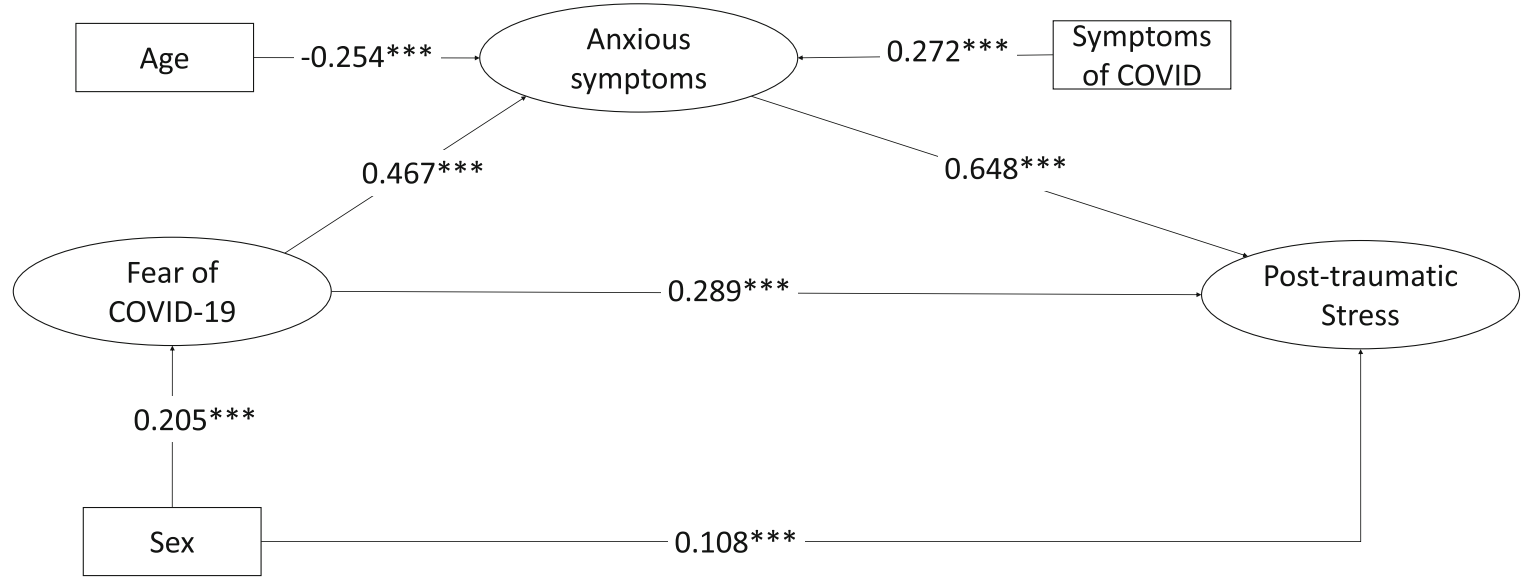

\section{Framework explaining Depression (model D)}

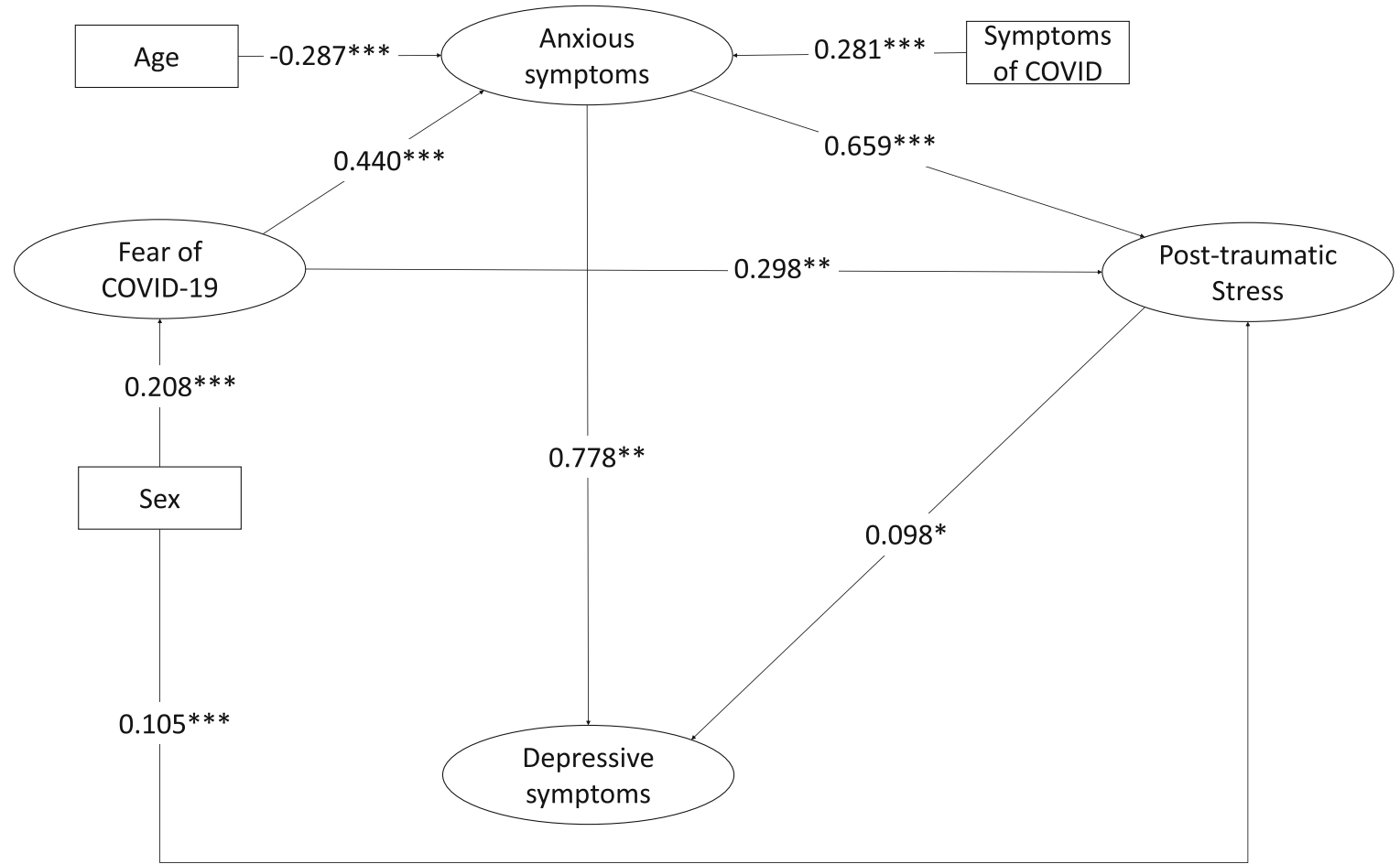

Fig. 2 Model of the relationship of fear of COVID-19, anxiety, and post-traumatic stress (model A) and the framework explaining depression (model D) for all participants $(n=830)$. Note: ${ }^{*} p<0.05,{ }^{* *} p<0.01,{ }^{* * *} p<0.001$

of health-care workers and the general population in the genesis of depressive symptoms.

Analysis of the triad of fear-anxiety-stress to predict depressive symptoms identified that only anxiety is strongly associated with depressive symptoms, as
PTSD has a small effect, and there is no direct relationship with fear with COVID-19. However, our study was supported by the fact that this triad mechanism represents a joint response to emotionally charged events such as a pandemic. Therefore, we 


\section{Relationship of fear, anxiety, and PTSD (model A)}
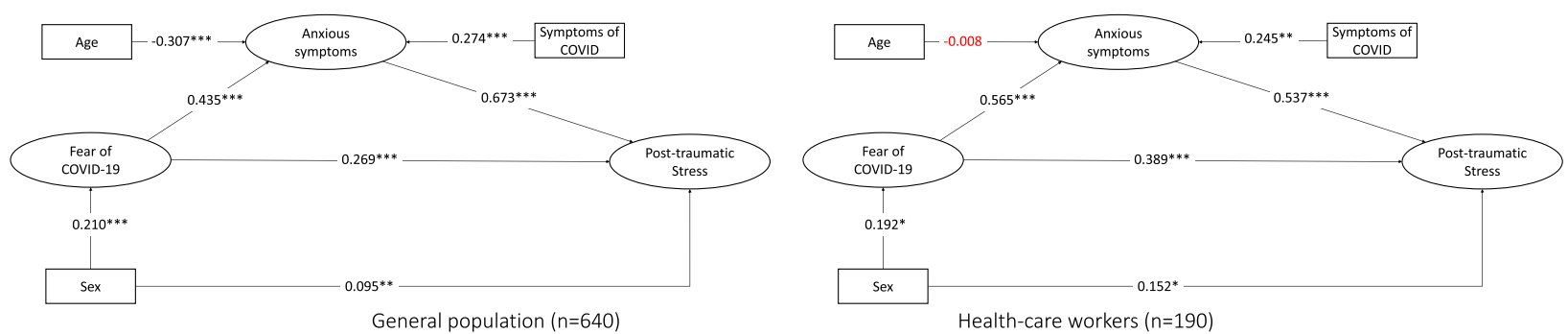

Framework explaining Depression (model D)
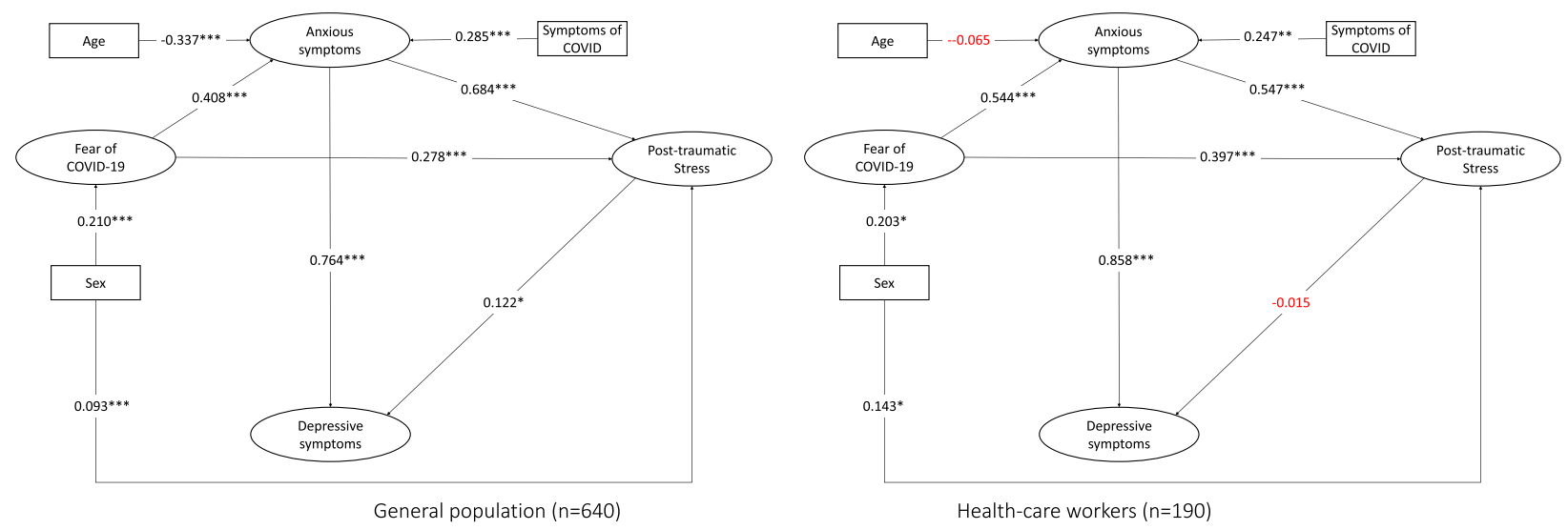

Fig. 3 Model of the relationship of fear of COVID-19, anxiety, and post-traumatic stress (model A) and the framework explaining depression (model D) for the general population and health-care workers. Note: ${ }^{*} p<0.05,{ }^{* *} p<0.01,{ }^{* * *} p<0.001$. Values in red are not significant

consider that its assessment as a whole (fear, anxiety, and stress) may better explain the presence of depressive symptoms.

Also, there is a higher prevalence of depressive, anxious, and stress symptoms in the general population than in health workers. Therefore, it is necessary to focus on mental health interventions and prevention activities for both groups.

\section{Contrasting findings with existing literature Prevalence}

In a previous study to compare the emotional effects of COVID-19 among three different groups in Peru was found a prevalence of clinically relevant symptoms of depression in general population (21\%), healthcare workers (9\%), and healthcare workers in COVID-19 areas $(8 \%)$ [40]. The prevalence recorded in the systematic review studies was higher than the values recorded in our research for depression (33.7 to 22.8\%), anxiety (29.6 to $23.2 \%$ ), and post-traumatic stress (31.9\%) [3, 41, 42]. However, it should be considered that the methods used to assess prevalence in systematic reviews are heterogeneous, belong to different times of the pandemic, and mostly correspond to high-income countries. Therefore, these elements could be overestimating the values.

The prevalence of each variable reported in our study is higher than those recorded in national studies conducted in Peru before the pandemic, where the prevalence of depression was $6.4 \%$ [43]. Although the pre-pandemic prevalence of depression in Peru is estimated to have been stable and not increasing [44], the national prevalence has likely increased during the pandemic. The increase in the prevalence is especially true for people who are aware of having a chronic disease [45], as they are at-risk populations.

\section{Structural equation model and relationship between variables}

Only two studies have been identified that pose predictive models that include fear of COVID-19, anxiety, stress, and depressive symptoms. This gap is understandable due to the limited number of published and pre-print studies as the pandemic, along with its related factors, is an emerging issue. 
The first study was carried out on Ecuadorian university students and proposed a model that predicts the depressive symptoms, from the fear of the COVID-19, anxiety, and stress [46]. This study agrees with our findings, and although the model presented is not the same as ours, it reinforces the hypothesis that the triad of fear, anxiety and stress predicts depressive symptoms. However, this study has two significant limitations. First, the instrument used to measure the main outcome is the Depression Anxiety Stress Scales (DASS), which presents good performance when used as a bifactorial instrument (a global dimension), but its performance presents inconsistencies when used as a three-dimensional correlated instrument (original DASS model) [47]. This inconsistency could introduce biases in the measurement of the main outcome. Second, the study does not evaluate other possible predictive models that could have a better fit. Furthermore, it is not clear what the process to define the model presented was. For example, it is not justified because the fear of COVID-19 and stress are not related, and studies have identified a strong relationship between both variables [48, 49].

The other study carried out in pregnant women evaluates a model where fear and anxiety related to COVID-19 predict the appearance of mental health problems, which were evaluated with the DASS but considering only an overall score that adds up the scores of anxiety, depression, and stress [50]. Although not directly comparable, this study identifies that fear of COVID-19 plays an essential role in the presence of mental health problems.

Some studies have been identified that partially analyze our proposed model. One study finds that fear of COVID19 has an indirect effect on the presence of depressive symptoms [51], and another study in general population identified that fear of COVID-19 has an association with mental health problems (i.e., anxiety and depression) [52], which supports our conclusions. Also, another study has reported a strong relationship between anxiety, stress, and depression in the context of the COVID-19 pandemic [49].

Other studies have evaluated variables that were not included in the study but may have a relevant role in the triad of fear, anxiety, and post-traumatic stress with depression. Two studies have evaluated the mediated role that uncertainty tolerance may play with COVID-19 fear and depressive symptoms $[53,54]$. Although this may be a limitation, our study evaluates in a more complex way the role that anxiety and stress have, unlike the mentioned studies. Furthermore, the mediating role of anxiety within the relationship between fear of COVID-19 and depression is not considered in the mentioned studies.

The proposed model shows a different behavior between health professionals and the general population. On the one hand, different studies have identified that age is negatively associated with anxious symptoms in the general population and is more frequent in younger people $[55,56]$ and that the general population has higher levels of anxiety and depression than health professionals [57]. A possible explanation for the decrease in the strength of the relationship between age and anxiety symptoms is that health care workers are exposed to less uncertainty about the virus (because of their biomedical training) and that they are better able to dissipate false news about COVID-19, which occurred at the beginning of the pandemic. On the other hand, the association between post-traumatic stress and depressive symptoms was not significant in health care workers but was significant in the general population. We have identified two possible explanations. First, although health care workers have had greater exposure to the virus on average, their biomedical training could be a factor that helps them to reduce their uncertainty about the virus and therefore have less stress [53]. Second, the fact of working itself is a factor that is associated with a greater perception of self-efficacy, and this is a protective factor for mental health problems such as stress or depression [52]. Both elements could be reducing the strength of the association between post-traumatic stress and the presence of depressive symptoms. In contrast, the general population, because they are on average not working, do not have a basic biomedical education, may experience greater uncertainty, have difficulty differentiating false news related to the virus, and thus experience more post-traumatic stress and depressive symptoms.

\section{Implications in public health and making decisions}

Previous studies show the key role that organizations and public health bodies play in promoting adaptive coping and reducing health worries and the emotional and psychological distress caused by the pandemic. Evidence highlights particular groups at risk of developing mental health problems (contact with infected patients, having children), and time points where risk may increase (initial response phase, when quarantined) [58]. Our study raises three main implications for public and global health in Peru and other Low and Middle-Income Countries (LMIC) with similar characteristics. First, the high prevalence of mental health problems recorded during the pandemic [3] makes it necessary to establish national policies and strategies for screening and epidemiological surveillance of the components of the triad of fear of COVID-19, anxiety, and post-traumatic stress, since these three elements predict the presence of depressive symptoms and other emotional problems. Health-care workers from Peru and other LMICs are overburdened by the pandemic and are in a fragmented health system [59]. It is recommended that technological tools such as apps or short ( 2 or 4 items) virtual selfreporting systems be used to collect information on 
emotional problems (i.e., anxiety, stress, fear, or depression) from the general population and health workers. These strategies have proven useful for addressing mental health needs and referring users with severe emotional problems in other countries during a pandemic emergency. Second, it is recommended to develop and implement preventive activities focused on the three elements of the triad (fear of COVID-19, anxiety, and posttraumatic stress). It is not only necessary to provide care to people who have moderate or severe mental health problems (depression, anxiety, or post-traumatic stress) but also to develop strategies aimed at people with mild and non-specific mental health indicators such as stress or fear of COVID-19 [60]. These strategies could prevent the latter individuals from evolving to a more advanced stage involving significant health problems and generating years of life lost due to disability [60]. Third, in Peru and other LMICs, the treatment gap for mental health problems such as depression is high, and it is estimated that only 1 in 10 people who require treatment receive it [44]. This gap may have increased during the pandemic, so actions to increase access to appropriate care need to be targeted. This model can be used to identify cases with a high fear of COVID-19 and facilitate their access to the health care system so that cases with greater severity of depression do not develop in the future.

At the level of mental health decision-makers, increased resources in mental health care are strongly recommended. In Peru, only $2 \%$ of all GDP is allocated to health, and only $0.2 \%$ of GDP is allocated to mental health [61], thus requiring increased human, financial, and political capital resources to improve mental health during and after the COVID-19 pandemic.

\section{Strengths and limitations}

Our study presents and assesses different models for understanding depressive symptoms using the triad of fear, anxiety, and stress. Our model applies a solid framework on the influence of this triad on mental health and applies it in the presence of depressive symptoms [69]. Another strong point of the study is the use of statistical methods that consider all these variables within their analysis. However, our study is not free of limitations. First, this cross-sectional study was conducted during the beginning of the first wave of COVID-19 in Peru (April 2020), so this model could vary during the later stages of the pandemic. Second, the data are from an urban city with the highest population density in the country (Lima, the capital of Peru), so the results could vary in a rural or less densely populated context (risk of infection). Third, the prevalence of depressive, anxious, and stress symptoms was evaluated with validated psychometric instruments, but this evaluation is not a substitute for clinical assessment, so misclassification can be a problem. Fourth, the scale for measuring PTSD (IES-R) is based on the criteria of the DMS-IV, which proposes three dimensions (intrusion, avoidance, and hyperactivity). However, the criteria for defining PTSD is modified in the DSM-5 where four dimensions are proposed (Reexperiencing, Avoidance, Negative Alterations in Cognitions and Mood, Hyperarousal), therefore, it is possible that the PTSD construct is being partially measured. This is not the case in the instruments used to measure depression and anxiety since the DMS-IV and DSM-5 criteria are equivalent. Fifth, other variables that could be useful to explain the model such as intolerance of uncertainty [53], pregnancy in the female participants [50], anxiety caused by COVID-19 [50], or income level [16] could not be included. Sixth, our study is a cross-sectional study so that causality statements cannot be made only about the association. Seventh, no differentiation was made between front-line staff working directly with COVID19 patients and other types of health professionals who may be doing remote work. Thus, it is possible that not all health care workers had the same level of exposure to the virus.

\section{Conclusions and recommendations}

Our study concludes that the triad of fear, anxiety and post-traumatic stress may explain more than $70 \%$ of depressive symptoms in the general population and health care workers during the COVID-19 pandemic. Also, a higher prevalence of depressive, anxious, and stressful symptoms is identified in the general population than in health care workers.

Researchers and decision-makers are encouraged to develop and implement policies and strategies to conduct screening for and epidemiological surveillance of fear of COVID-19, anxiety symptoms, and stress, as these variables, are predecessors to depressive symptoms. Another recommendation is the development and implementation of preventive actions for these three elements (fear, anxiety, and stress) in the general population and health-care workers so that the prevalence of depressive symptoms can be reduced.

\section{Acknowledgments}

ALVE thanks DR for his valuable contribution in reviewing the language of the manuscript.

\section{Authors' contributions}

David Villarreal-Zegarra: Conceived and designed the analysis; contributed data or analysis tools; performed the analysis; wrote the paper. Anthony Copez-Lonzoy: Conceived and designed the analysis; contributed data or analysis tools; performed the analysis; wrote the paper. Ana L. Vilela-Estrada: Conceived and designed the analysis; wrote the paper; supervision of the paper. Jeff Huarcaya-Victoria: Conceived and designed the analysis; collected 
the data; wrote the paper; supervision of the paper. The authors read and approved the final manuscript.

\section{Funding}

The study was self-financed.

\section{Availability of data and materials}

The database is available from https://doi.org/10.6084/m9.figshare.13683955

\section{Declarations}

\section{Ethics approval and consent to participate}

The study protocol and the instruments used for the evaluation were approved by the ethics committee of the Universidad San Martin de Porres (Oficio No. 227-2020-CIEI-FMH-USMP). All procedures performed in this study involving human participants were conducted according to the research team's organizational Ethics Board and the 1975 Helsinki Declaration. Participants voluntarily agreed to participate in the study, and informed consent was obtained from all participants. The data collected were anonymous and confidential so that the study did not involve any risk for the participants.

\section{Consent for publication}

Not applicable.

\section{Competing interests}

The authors have declared that no competing interests exist.

\section{Author details}

${ }^{1}$ Escuela de Medicina, Universidad César Vallejo, Trujillo, Peru. ${ }^{2}$ Instituto Peruano de Orientación Psicológica, Lima, Peru. ${ }^{3}$ Unidad de Investigación en Bibliometría, Universidad San Ignacio de Loyola, Lima, Peru. ${ }^{4}$ Asociación Peruana de Profesionales de las Adicciones, Lima, Peru. ${ }^{5}$ CRONICAS Center of Excellence in Chronic Diseases, Universidad Peruana Cayetano Heredia, Lima, Peru. ${ }^{6}$ Departamento de Psiquiatría, Hospital Nacional Guillermo Almenara Irigoyen, Lima, Peru. ${ }^{7}$ Departamento Académico de Psiquiatría, Universidad Nacional Mayor de San Marcos, Lima, Peru.

Received: 19 January 2021 Accepted: 14 August 2021

\section{Published online: 17 September 2021}

\section{References}

1. Sala Situacional COVID-19 Perú [https://covid19.minsa.gob.pe/sala_situa cional.asp]. Accessed 6 June 2021.

2. Huremovic D. Social distancing, quarantine, and isolation. In: Huremovic D, editor. Psychiatry of pandemics a mental health response to infection outbreak. Switzerland: Springer; 2019. p. 85-94.

3. Salari N, Hosseinian-Far A, Jalali R, Vaisi-Raygani A, Rasoulpoor $\mathrm{S}$, Mohammadi $\mathrm{M}$, et al. Prevalence of stress, anxiety, depression among the general population during the COVID-19 pandemic: a systematic review and meta-analysis. Glob Health. 2020;16(1):57. https://doi.org/10.1186/s12 992-020-00589-w.

4. Brooks SK, Webster RK, Smith LE, Woodland L, Wessely S, Greenberg N, et al. The psychological impact of quarantine and how to reduce it: rapid review of the evidence. Lancet. 2020;395(10227):912-20.

5. Huarcaya-Victoria J, Villarreal-Zegarra D, Podestà A, Luna-Cuadros MA. Psychometric properties of a Spanish version of the fear of COVID-19 scale in general population of Lima, Peru. Int J Ment Health Addict. 2020:1-14. https://doi.org/10.1007/s11469-020-00354-5.

6. Greenwood BN, Thompson RS, Opp MR, Fleshner M. Repeated exposure to conditioned fear stress increases anxiety and delays sleep recovery following exposure to an acute traumatic stressor. Front Psychiatry. 2014;5: 146. https://doi.org/10.3389/fpsyt.2014.00146.

7. Lutz B, Marsicano G, Maldonado R, Hillard CJ. The endocannabinoid system in guarding against fear, anxiety and stress. Nat Rev Neurosci. 2015;16(12): 705-18. https://doi.org/10.1038/nrn4036.

8. Ding J, Han F, Wen L, Xiao B, Shi Y. The role of $\beta$-arrestin-2 on fear/anxiousrelated memory in a rat model of post-traumatic stress disorder. J Affect Disord. 2017;213:1-8. https://doi.org/10.1016/j.jad.2016.12.043.

9. Forbes D, Parslow R, Creamer M, O'Donnell M, Bryant R, McFarlane A, et al. A longitudinal analysis of posttraumatic stress disorder symptoms and their relationship with fear and anxious-misery disorders: implications for DSM-V. J Affect Disord. 2010;127(1-3):147-52. https:/doi.org/10.1016/j.jad.2010.05.005.

10. Andrews G, Charney DS, Sirovatka PJ, Regier DA. Stress-induced and fear circuitry disorders: refining the research agenda for DSM-V. USA: American Psychiatric Pub; 2009.

11. Siebenhaar KU, Köther AK, Alpers GW. Dealing with the COVID-19 Infodemic: distress by information, information avoidance, and compliance with preventive measures. Front Psychol. 2020;11:567905. https://doi.org/1 0.3389/fpsyg.2020.567905.

12. Kim YJ. Posttraumatic stress disorder as a mediator between trauma exposure and comorbid mental health conditions in north Korean refugee youth resettled in South Korea. J Interpers Viol. 2016;31(3):425-43. https:// doi.org/10.1177/0886260514555864

13. An Y, Huang J, Chen Y, Deng Z. Longitudinal cross-lagged relationships between posttraumatic stress disorder and depression in adolescents following the Yancheng tornado in China. Psychol Trauma. 2019;11(7):7606. https://doi.org/10.1037/tra0000455.

14. Ni MY, Jiang C, Cheng KK, Zhang W, Gilman SE, Lam TH, et al. Stress across the life course and depression in a rapidly developing population: the Guangzhou biobank cohort study. Int J Geriatr Psychiatry. 2016;31(6):629-37. https://doi.org/10.1002/gps.4370.

15. Sharafkhaneh A, Yohannes AM, Hanania NA, Kunik ME. Depression and anxiety in patients with chronic respiratory diseases. New York: Springer; 2017. https://doi.org/10.1007/978-1-4939-7009-4.

16. Rehman U, Shahnawaz MG, Khan NH, Kharshiing KD, Khursheed M, Gupta K, et al. Depression, anxiety and stress among Indians in times of Covid-19 lockdown. Community Ment Health J. 2020;57(1):1-7. https://doi.org/10.1 007/s10597-020-00664-X

17. Hair JF, Anderson RE, Tatham RL, Black WC. Análisis multivariante, vol. 491. España: Prentice Hall Madrid; 1999.

18. Malaeb D, Salameh P, Barbar S, Awad E, Haddad C, Hallit R, et al. Problematic social media use and mental health (depression, anxiety, and insomnia) among Lebanese adults: any mediating effect of stress? Perspectives in psychiatric care; 2020.

19. Park J, Elbert T, Kim SJ, Park J. The contribution of posttraumatic stress disorder and depression to insomnia in north Korean refugee youth. Front Psychiatry. 2019;10:211. https://doi.org/10.3389/fpsyt.2019.00211.

20. Subica AM, Claypoole KH, Wylie AM. PTSD'S mediation of the relationships between trauma, depression, substance abuse, mental health, and physical health in individuals with severe mental illness: evaluating a comprehensive model. Schizophr Res. 2012;136(1-3):104-9. https://doi.org/10.1016/j.schres.2011.10.018.

21. lacobucci D. Structural equations modeling: fit indices, sample size, and advanced topics. J Consum Psychol. 2010;20(1):90-8. https://doi.org/10.101 6/j.jcps.2009.09.003.

22. Ahorsu DK, Lin CY, Imani V, Saffari M, Griffiths MD, Pakpour AH. The fear of COVID-19 scale: development and initial validation. Int J Ment Health Addict. 2020:1-9. https://doi.org/10.1007/s11469-020-00270-8.

23. Weiss DS, et al. The impact of event scale-Revised. In: Wilson JP, Keane TM, editors. Assessing psychological trauma and PTSD: a practitioner's handbook. New York: Guilford Press; 1997. p. 399-411.

24. Guest R, Tran Y, Gopinath B, Cameron ID, Craig A. Prevalence and psychometric screening for the detection of major depressive disorder and post-traumatic stress disorder in adults injured in a motor vehicle crash who are engaged in compensation. BMC Psychol. 2018;6(1):4. https://doi.org/1 0.1186/s40359-018-0216-5.

25. Spitzer RL, Kroenke K, Williams JB. Validation and utility of a self-report version of PRIME-MD: the PHQ primary care study. JAMA. 1999;282(18): 1737-44. https://doi.org/10.1001/jama.282.18.1737.

26. Manea L, Gilbody S, McMillan D. A diagnostic meta-analysis of the patient health Questionnaire-9 (PHQ-9) algorithm scoring method as a screen for depression. Gen Hosp Psychiatry. 2015;37(1):67-75. https://doi.org/10.1016/j. genhosppsych.2014.09.009.

27. Villarreal-Zegarra D, Copez-Lonzoy A, Bernabe-Ortiz A, Melendez-Torres GJ, Bazo-Alvarez JC. Valid group comparisons can be made with the patient health questionnaire (PHQ-9): a measurement invariance study across groups by demographic characteristics. PLoS One. 2019;14(9):e0221717. https://doi.org/10.1371/journal.pone.0221717.

28. Spitzer RL, Kroenke K, Williams JW, Löwe B. A brief measure for assessing generalized anxiety disorder: the GAD-7. Arch Intern Med. 2006;166(10): 1092-7. https://doi.org/10.1001/archinte.166.10.1092. 
29. Plummer F, Manea L, Trepel D, McMillan D. Screening for anxiety disorders with the GAD-7 and GAD-2: a systematic review and diagnostic metaanalysis. Gen Hosp Psychiatry. 2016;39:24-31. https://doi.org/10.1016/j. genhosppsych.2015.11.005

30. García-Campayo J, Zamorano E, Ruiz MA, Pardo A, Pérez-Páramo M, LópezGómez V, et al. Cultural adaptation into Spanish of the generalized anxiety disorder-7 (GAD-7) scale as a screening tool. Health Qual Life Outcomes. 2010;8(1):8. https://doi.org/10.1186/1477-7525-8-8.

31. Palomino-Oré C, Huarcaya-Victoria J. Trastornos por estrés debido a la cuarentena durante la pandemia por la COVID-19. J Horizonte Médico. 2020;20:e1218. https://doi.org/10.24265/horizmed.2020.v20n4.10.

32. Wang C, Pan R, Wan X, Tan Y, Xu L, Ho CS, et al. Immediate Psychological Responses and Associated Factors during the Initial Stage of the 2019 Coronavirus disease (COVID-19) epidemic among the general population in China. Int J Environ Res Public Health. 2020;17(5). https://doi.org/10.3390/ ijerph17051729.

33. Kelley K, Pornprasertmanit S. Confidence intervals for population reliability coefficients: evaluation of methods, recommendations, and software for composite measures. Psychol Methods. 2016;21(1):69-92. https://doi.org/1 $0.1037 / a 0040086$.

34. Brown TA. Confirmatory factor analysis for applied research. 2nd ed. New York: The Guilford Press; 2015.

35. Dominguez-Lara SA. ¿ Matrices policóricas/tetracóricas o matrices Pearson? Un estudio metodológico. Revista Argentina de Ciencias del Comportamiento. 2014;6(1):39-48.

36. Timmerman ME, Lorenzo-Seva U. Dimensionality assessment of ordered polytomous items with parallel analysis. Psychol Methods. 2011;16(2):209_ 20. https://doi.org/10.1037/a0023353.

37. González-Rivera J, Rosario-Rodríguez A, Cruz-Santos A. Escala de Ansiedad por Coronavirus: Un Nuevo Instrumento para Medir Síntomas de Ansiedad Asociados al COVID-19. Interacciones. 2020;6(3):e163.

38. Hu L-T, Bentler PM. Fit indices in covariance structure modeling: sensitivity to underparameterized model misspecification. Psychol Methods. 1998;3(4): 424-53. https://doi.org/10.1037/1082-989X.3.4.424.

39. Ferguson CJ. An effect size primer: a guide for clinicians and researchers. Prof Psychol Res Pract. 2009:40(5):532-8.

40. Pedraz-Petrozzi B, Krüger-Malpartida H, Arevalo-Flores M, Salmavides-Cuba F, Anculle-Arauco V, Dancuart-Mendoza M. Emotional impact on health personnel, medical students, and general population samples during the COVID-19 pandemic in Lima, Peru. Rev Colomb Psiquiatr. 2021;50(3):189-98. https://doi.org/10.1016/j.rcp.2021.04.006.

41. Luo M, Guo L, Yu M, Jiang W, Wang $H$. The psychological and mental impact of coronavirus disease 2019 (COVID-19) on medical staff and general public - a systematic review and meta-analysis. Psychiatry Res. 2020;291: 113190. https://doi.org/10.1016/j.psychres.2020.113190.

42. Pappa S, Ntella V, Giannakas T, Giannakoulis VG, Papoutsi E, Katsaounou P. Prevalence of depression, anxiety, and insomnia among healthcare workers during the COVID-19 pandemic: a systematic review and metaanalysis. Brain Behav Immun. 2020;88:901-7. https://doi.org/10.1016/j. bbi.2020.05.026

43. Hernández-Vásquez A, Vargas-Fernández R, Bendezu-Quispe G, Grendas LN. Depression in the Peruvian population and its associated factors: analysis of a national health survey. J Affect Disord. 2020;273:291-7. https://doi.org/10.1 016/j.jad.2020.03.100.

44. Villarreal-Zegarra D, Cabrera-Alva M, Carrillo-Larco RM, Bernabe-Ortiz A Trends in the prevalence and treatment of depressive symptoms in Peru: a population-based study. BMJ Open. 2020;10(7):e036777. https://doi.org/1 0.1136/bmjopen-2020-036777.

45. Villarreal-Zegarra D, Bernabe-Ortiz A. Association between arterial hypertension and depressive symptoms: results from population-based surveys in Peru. Asia Pac Psychiatry. 2020;12(2):e12385. https://doi.org/1 $0.1111 / a p p y .12385$

46. Rodríguez-Hidalgo AJ, Pantaleón Y, Dios I, Falla D. Fear of COVID-19, Stress, and Anxiety in University Undergraduate Students: A Predictive Model for Depression. Front Psychol. 2020;11:591797. https://doi.org/10.3389/fpsyg.202 0.591797 .

47. Lee J, Lee EH, Moon SH. Systematic review of the measurement properties of the depression anxiety stress Scales-21 by applying updated COSMIN methodology. Qual Life Res. 2019;28(9):2325-39. https://doi.org/10.1007/ s11136-019-02177-x
48. Blanco-Donoso LM, Moreno-Jiménez J, Amutio A, Gallego-Alberto L, Moreno-Jiménez B, Garrosa E. Stressors, job resources, fear of contagion, and secondary traumatic stress among nursing home Workers in Face of the COVID-19: the case of Spain. J Appl Gerontol. 2020;(3):244-56. https:// doi.org/10.1177/0733464820964153.

49. Tayyib NA, Alsolami FJ. Measuring the extent of stress and fear among registered nurses in KSA during the COVID-19 outbreak. J Taibah Univ Med Sci. 2020;15(5):410-6. https://doi.org/10.1016/j.jtumed.2020.07.012.

50. Salehi L, Rahimzadeh M, Molaei E, Zaheri H, Esmaelzadeh-Saeieh S. The relationship among fear and anxiety of COVID-19, pregnancy experience, and mental health disorder in pregnant women: a structural equation model. Brain Behav. 2020;10(11):e01835. https://doi.org/10.1002/brb3.1835.

51. Mahmud MS, Talukder MU, Rahman SM. Does 'Fear of COVID-19' trigger future career anxiety? An empirical investigation considering depression from COVID-19 as a mediator. Int J Soc Psychiatry. 2020;(1):35-45. https:// doi.org/10.1177/0020764020935488.

52. Boluarte-Carbajal A, Navarro-Flores A, Villarreal-Zegarra D. Explanatory model of perceived stress in the general population: a cross-sectional study in Peru during the COVID-19 context. Front Psychol. 2021;12:673945. https://doi. org/10.3389/fpsyg.2021.673945.

53. Bakioğlu F, Korkmaz O, Ercan H. Fear of COVID-19 and positivity: mediating role of intolerance of uncertainty, depression, anxiety, and stress. Int J Ment Health Addict. 2020:1-14. https://doi.org/10.1007/s11469-020-00331-y.

54. Voitsidis P, Nikopoulou VA, Holeva V, Parlapani E, Sereslis K, Tsipropoulou V, et al. The mediating role of fear of COVID-19 in the relationship between intolerance of uncertainty and depression. Psychol Psychother. 2020;94(3): 884-93. https://doi.org/10.1111/papt.12315.

55. Solomou I, Constantinidou F. Prevalence and Predictors of Anxiety and Depression Symptoms during the COVID-19 Pandemic and Compliance with Precautionary Measures: Age and Sex Matter. Int J Environ Res Public Health. 2020;17(14). https://doi.org/10.3390/ijerph17144924.

56. Nwachukwu I, Nkire N, Shalaby R, Hrabok M, Vuong W, Gusnowski A, et al. COVID-19 Pandemic: Age-Related Differences in Measures of Stress, Anxiety and Depression in Canada. Int J Environ Res Public Health. 2020;17(17). https://doi.org/10.3390/ijerph17176366.

57. Muhammad Alfareed Zafar S, Junaid Tahir M, Malik M, Irfan Malik M, Kamal Akhtar F, Ghazala R. Awareness, anxiety, and depression in healthcare professionals, medical students, and general population of Pakistan during COVID-19 Pandemic: A cross sectional online survey. Med J Islam Repub Iran. 2020;34:131. https://doi.org/10.47176/mjiri.34.131.

58. Waring S, Giles S. Rapid evidence assessment of mental health outcomes of pandemics for health care workers: implications for the Covid-19 pandemic. Front Public Health. 2021;9:629236. https://doi.org/10.3389/fpubh.2021.62 9236.

59. Ministerio de Salud. Plan Nacional de Fortalecimiento de Servicios de Salud Mental Comunitaria 2018-2021. Perú: Ministerio de Salud; 2018.

60. Patel V, Saxena S, Lund C, Thornicroft G, Baingana F, Bolton P, et al. The Lancet Commission on global mental health and sustainable development. Lancet. 2018;392(10157):1553-98.

61. Cavero V, Hidalgo L, Rivera D. Perfil de País: Perú - Análisis para una campaña y defensa de la salud mental. UK: Mental Health Innovation Network; 2020.

\section{Publisher's Note}

Springer Nature remains neutral with regard to jurisdictional claims in published maps and institutional affiliations.

\section{Ready to submit your research? Choose BMC and benefit from:}

- fast, convenient online submission

- thorough peer review by experienced researchers in your field

- rapid publication on acceptance

- support for research data, including large and complex data types

- gold Open Access which fosters wider collaboration and increased citations

- maximum visibility for your research: over $100 \mathrm{M}$ website views per year

At BMC, research is always in progress.

Learn more biomedcentral.com/submission 